\title{
Les phlébotomes de l'île de Chypre III - INVENTAIRE FAUNISTIQUE
}

\author{
DEPAQUIT J.*, LÉGER N.*, FERTÉ H.*, RIOUX J.A.**, GANTIER J.C.***, \\ MICHAELIDES A.**** \& ECONOMIDES P.****
}

Summary : PHLEBOTOMINE SANDFLES OF CYPRUS. III - SPECIES INVENTORY Three surveys on canine leishmaniasis were carried out in Cyprus (1993, 1998 and 1999) emphasise the presence of eleven species of phlebotomine sandflies

Phlebotomus (Phlebotomus) papatasi, P. (Paraphlebotomus) alexandri, P. (Pa.) jacusieli (first mention in Cyprus), P. (Pa.) sergenti, P. (Larroussius) galilaeus, P. (L.) tobbi, P. (Transphlebotomus) economidesi, P. (T.) mascittii, Sergentomyia (Sergentomyia) azizi (its specific statute is validated by the authors), S. (S.) fallax et S. (S.) minuta. P. (Adlerius) kyreniae was not caught during the surveys. The authors propose hypothesis of settlement of the island by phlebotomine sandflies according this species inventory. A first migration period took probably place during the Miocene time and a second one during the Pleistocene time.

KEY WORDS : phlebotomine sandflies, Phlebotomus, Sergentomyia, Cyprus inventory

\section{INTRODUCTION}

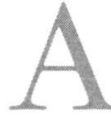

u cours de trois missions consacrées à l'étude de la transmission de la leishmaniose canine à Chypre en 1993 (16-26 août), 1998 (21-28 août) et 1999 (5-18 septembre), nous avons réuni une collection de plusieurs milliers de Phlébotomes grâce à laquelle nous avons pu compléter les inventaires réalisés par Adler (1946) et Minter \& Eitrem (1989).

\section{MATÉRIEL ET MÉTHODES}

L es captures ont été réalisées aux pièges CDC miniatures modifiés ${ }^{(1)}$. Quelques captures manuelles ont également été pratiquées. Tous ces piégeages ont eu lieu essentiellement autour des che-

* Faculté de Pharmacie, 51096 Reims Cedex, France.

*** Institut de Botanique, 34000 Montpellier, France.

**** Faculté de Pharmacie, 92290 Châtenay-Malabry, France.

**** Veterinary Services, Nicosia, Cyprus.

Correspondance : J. Depaquit, Laboratoire de Parasitologie, Faculté de Pharmacie, 51, rue Cognacq-Jay, 51096 Reims Cedex, France. Tél. : 0326913723 - Fax : 0326913597.

E-mail : jerome.depaquit@univ-reims.fr

(1) John W. Hock Company, Insect sampling devices for medical veterinary and agricultural entomology, P.O. box 12852, Gainesville, FL 32604, USA

\section{Résumé :}

Trois missions menées en 1993, 1998 et 1999 consacrées à l'étude de la transmission de la leishmaniose canine à Chypre ont permis la capture de plusieurs milliers de phlébotomes appartenant à onze espèces: Phlebotomus (Phlebotomus) papatasi,

P. (Paraphlebotomus) alexandri, P. (Pa.) jacusieli (première mention à Chypre), P. (Pa.) sergenti, P. (Larroussius) galilaeus, P. (L.) tobbi, P. (Transphlebotomus) economidesi, P. (T.) mascittii, Sergentomyia (Sergentomyia) azizi (dont les auteurs revalident le statut spécifique), S. (S.) fallax et S. (S.) minuta. P. (Adlerius) kyreniae n'a pas été retrouvé durant les prospections. Des hypothèses relatives au peuplement de l'île sont formulées Il'une au Miocène durant la crise messinienne, l'autre au Pléistocène) en accord avec cet inventaire faunistique.

MOTS CLÉS : phlébotomes, Phlebotomus, Sergentomyia, Chypre, inventaire.

nils dans les localités où des cas de leishmaniose canine avaient été signalés ainsi que dans divers biotopes sauvages éloignés des habitations (Léger et al., 2000b : Fig. 1).

Les femelles ont été disséquées extemporanément (recherche de leishmanies) et déterminées après montage de la tête et des spermathèques dans le liquide de Marc-André. Le reste des captures (mâles et femelles) a été conservé dans l'alcool à $95 \%$ et identifié ultérieurement après éclaircissement (potasse à $10 \%$, MarcAndré) et montage soit dans la gomme au chloral, soit, après déshydratation, dans le baume du Canada.

\section{RÉSULTATS}

T a liste des espèces mentionnées à Chypre d'après Adler (1946), Minter et Eitrem (1989) et nos propres captures est rapportée dans le tableau I.

\section{COMMENTAIRES SUR LES ESPÈCES}

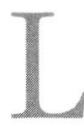
'inventaire réalisé est purement qualitatif : notre méthodologie de piégeage et d'utilisation des phlébotomes ne permet pas l'exploitation quantitative de l'ensemble des captures. 


\begin{tabular}{|c|c|c|}
\hline Présente étude & Adler & Minter \& Eitrem \\
\hline $\begin{array}{rr} & 1998- \\
1993 \quad 1999\end{array}$ & 1944 & $\begin{array}{l}1971- \\
1984\end{array}$ \\
\hline
\end{tabular}

\begin{tabular}{|c|c|c|c|c|c|}
\hline \multicolumn{6}{|c|}{ Genre Pblebotomus } \\
\hline \multicolumn{6}{|c|}{ sous-genre Pblebotomus } \\
\hline P. papatasi & + & + & + & + & + \\
\hline \multicolumn{6}{|c|}{ sous-genre Parapblebo- } \\
\hline \multicolumn{6}{|l|}{ tomus } \\
\hline P. sergenti & + & + & + & + & 0 \\
\hline P. jacusieli & + & + & 0 & 0 & 0 \\
\hline P. alexandri & + & + & + & 0 & 0 \\
\hline \multicolumn{6}{|c|}{ sous-genre Larroussius } \\
\hline P. tobbi & + & + & + & 0 & + \\
\hline P. galilaeus & + & + & $+(1)$ & + & + \\
\hline \multicolumn{6}{|c|}{ sous-genre Transphlebo- } \\
\hline \multicolumn{6}{|l|}{ tomus } \\
\hline P. mascittii & + & + & $+(2)$ & 0 & 0 \\
\hline P. economidesi & 0 & + & 0 & 0 & 0 \\
\hline \multicolumn{6}{|c|}{ sous-genre Adlerius } \\
\hline P. kyreniae & 0 & 0 & + & 0 & 0 \\
\hline \multicolumn{6}{|c|}{ Genre Sergentomyia } \\
\hline \multicolumn{6}{|c|}{ sous-genre Sergentomyia } \\
\hline S. minuta & + & + & + & + & + \\
\hline S. $a z i z i$ & + & + & + & 0 & $+(3)$ \\
\hline S. fallax & + & + & + & 0 & $+(4)$ \\
\hline
\end{tabular}

Tableau I. - Liste des espèces mentionnées à Chypre d'après Adler (1946), Minter et Eitrem (1989) et nos propres captures.

(1): mentionnée sous le nom de P.perfiliewi; (2) : mentionnée sous le nom de P. larroussei; (3) : mentionnée sous le nom de antennata group; (4) : mentionnée sous le nom de fallax group.

\section{PhleBOtOMUS PAPATASI (Scopoli, 1786) (fig. 1)}

Toujours abondante dans les pièges et dans les captures manuelles nocturnes, c'est la seule espèce capturée sur appât humain. Très anthropophile, elle semble présente dans les maisons où nous n'avons effectué que très peu de piégeages, et dans les villes de basse altitude où elle constitue à certaines périodes, une nuisance non négligeable.

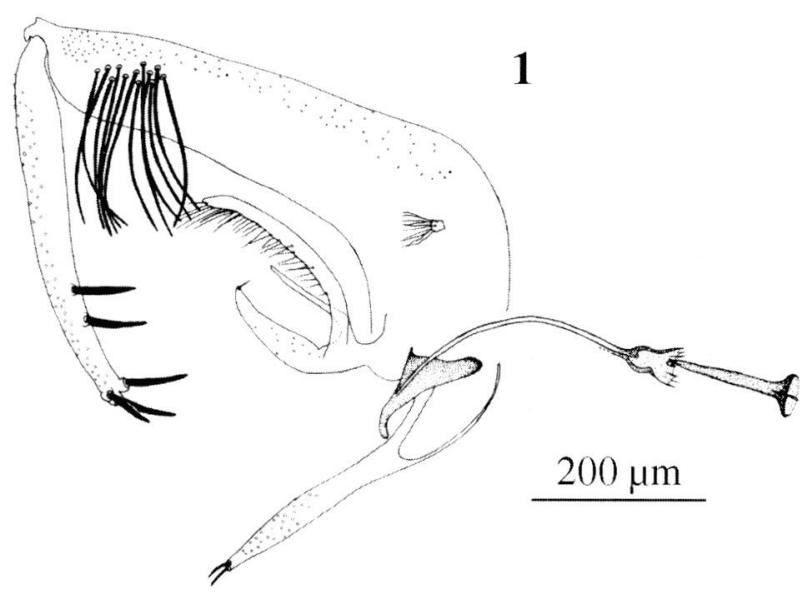

PhlEBOtomus ALEXANDRI SinTon, 1928 (fig. 2)

Non retrouvée par Minter et Eitrem, elle est signalée par Adler uniquement sur la côte kyrénienne, non prospectée par nous. Nos exemplaires (deux mâles et 21 femelles) proviennent des villages d'Agios Georgios au sud-est de l'île et d'Armenochori.

\section{Phlebotomus jacusieli Theodor, 1947 (fig. 3)}

Décrite de Palestine, cette espèce n'est signalée à Chypre ni par Adler en 1946 (mais elle n'a été décrite qu'en 1947), ni par Minter et Eitrem (1989). Nos exemplaires (trois mâles et neuf femelles) proviennent des villages de Monagri et de Pyrgos.

\section{Phlebotomus sergenti Parrot, 1917 (fig. 4)}

L'espèce présente à Chypre est bien $P$. sergenti s. str. et non $P$. similis, espèce voisine présente en Grèce (y compris à Rhodes et en Crète) et en Turquie jusqu'au Taurus au delà duquel elle est remplacée par $P$. sergent $i$ (Depaquit et al., 1998). Quelques variations au niveau de l'armature pharyngienne sont relevées. Elles sont de niveau intrapopulationnel. Des études moléculaires par PCR-RFLP menées sur quelques spécimens grecs et cypriotes n'ont pas mis en évidence de variabilité (Aransay et al., 1999). Cependant, un arbre phylétique construit sur la base des séquences de la sous-unité ribosomale 18S (Aransay et al., 2000) individualise deux rameaux terminaux indépendants, l'un correspondant à " $P$. sergenti " de Crète $(=P$. similis que les auteurs ignorent) et l'autre à $P$. sergenti de Chypre $(=P$. sergenti $\mathrm{s}$. str.). Cette différence interspécifique est confirmée par les séquences de l'ITS 2 qui individualisent nettement $P$. similis et $P$. sergenti (Depaquit et al., 2000).
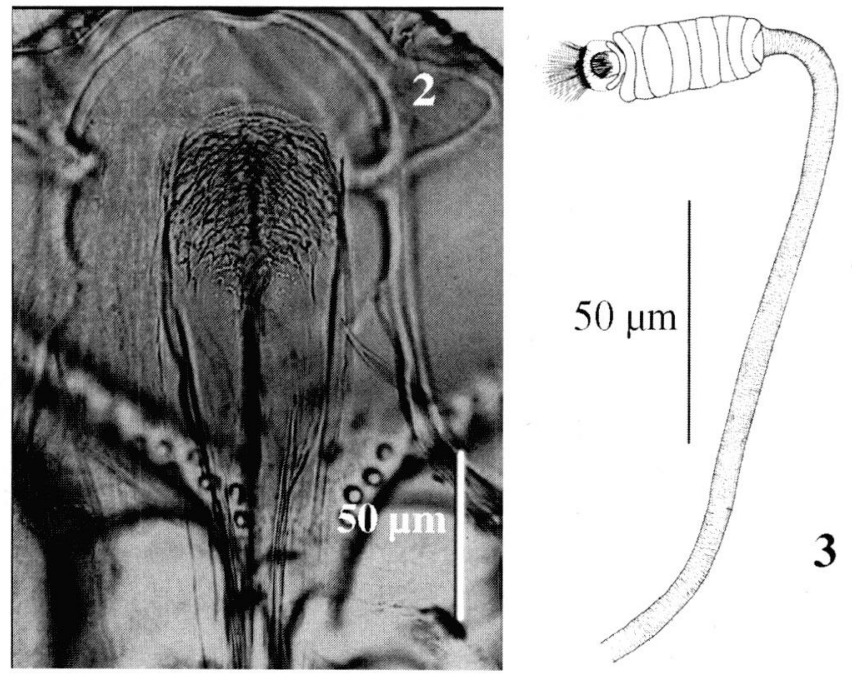

Fig. 1. - Phlebotomus papatasi. Mâle : génitalia (1). Femelle : pharynx (2) et spermathèque (3). 

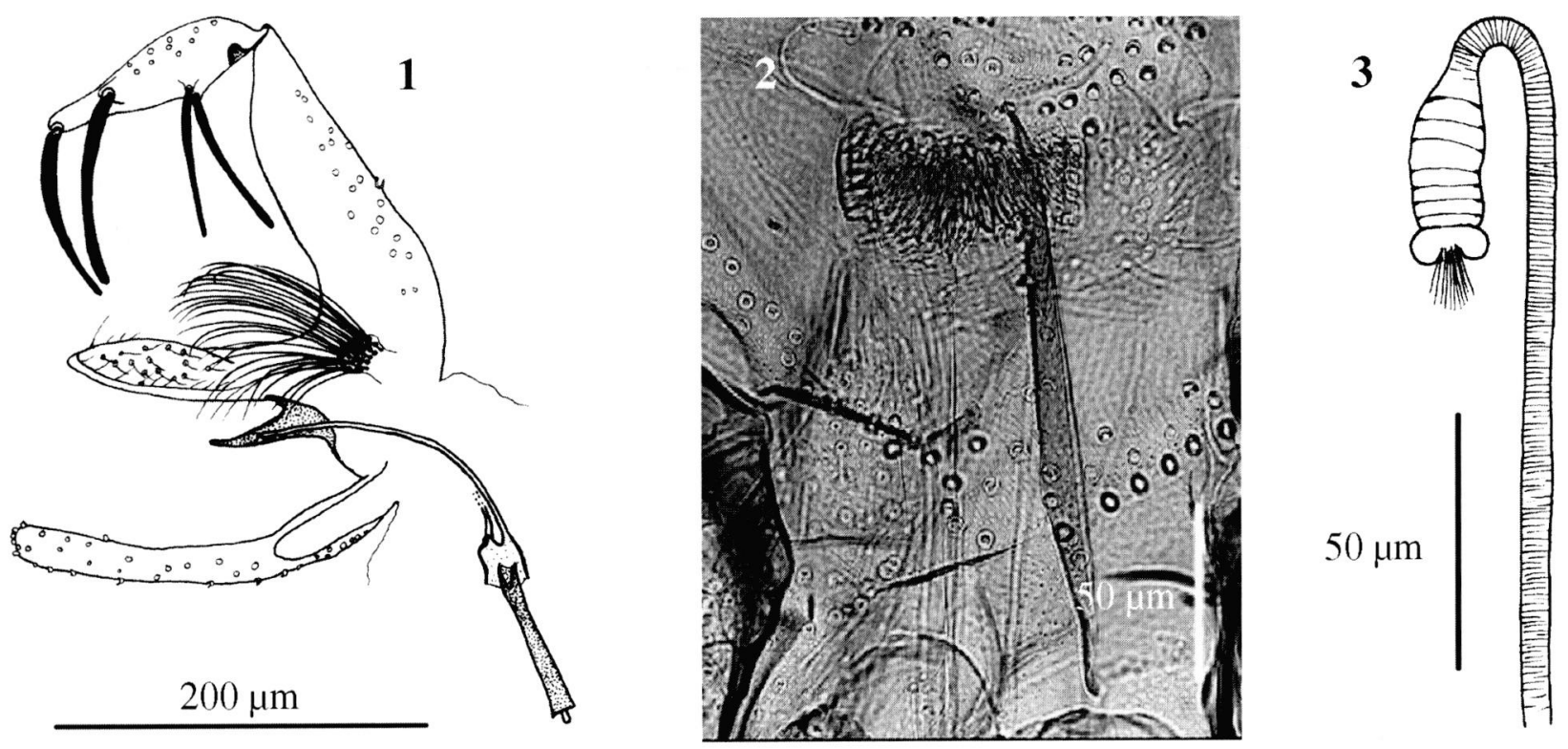

Fig. 2. - Pblebotomus alexandri. Mâle : génitalia (1). Femelle : pharynx (2) et spermathèque (3).
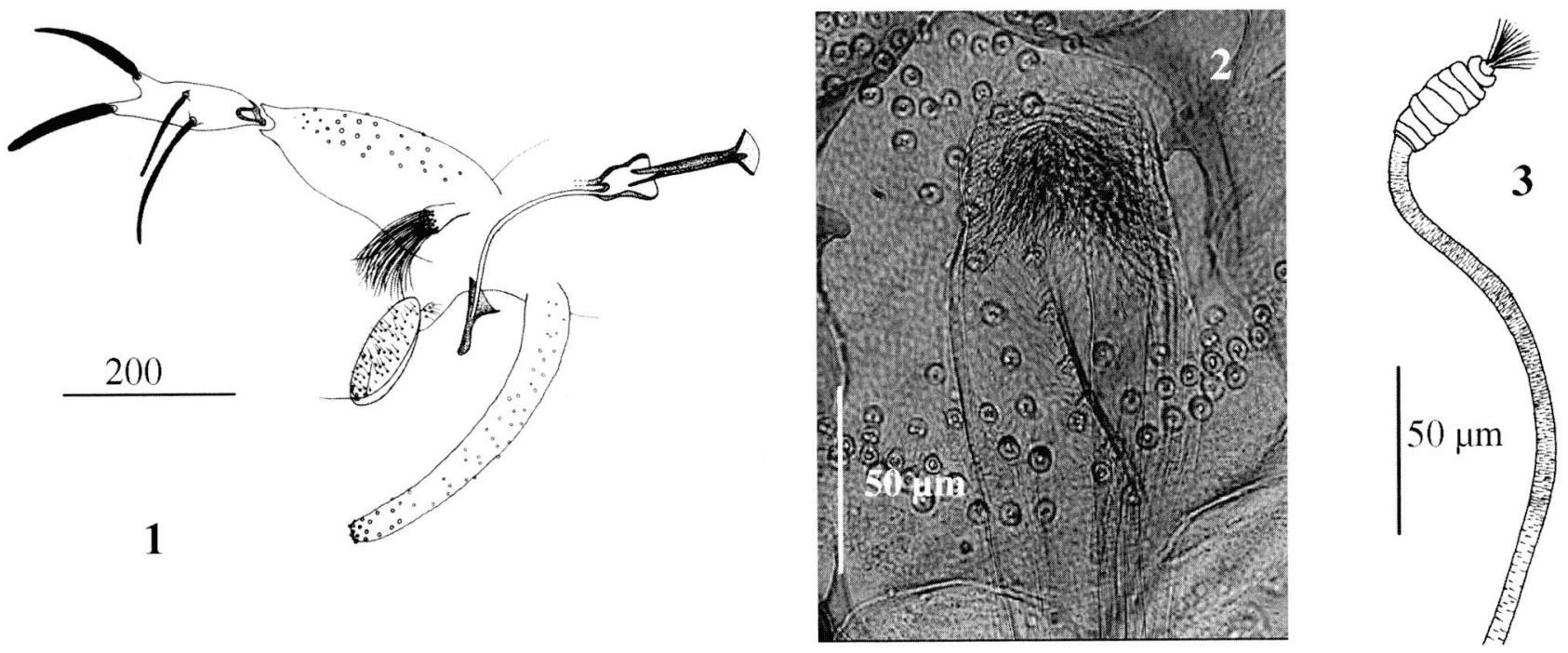

Fig. 3. - Pblebotomus jacusieli. Mâle : génitalia (1). Femelle : pharynx (2) et spermathèque (3).
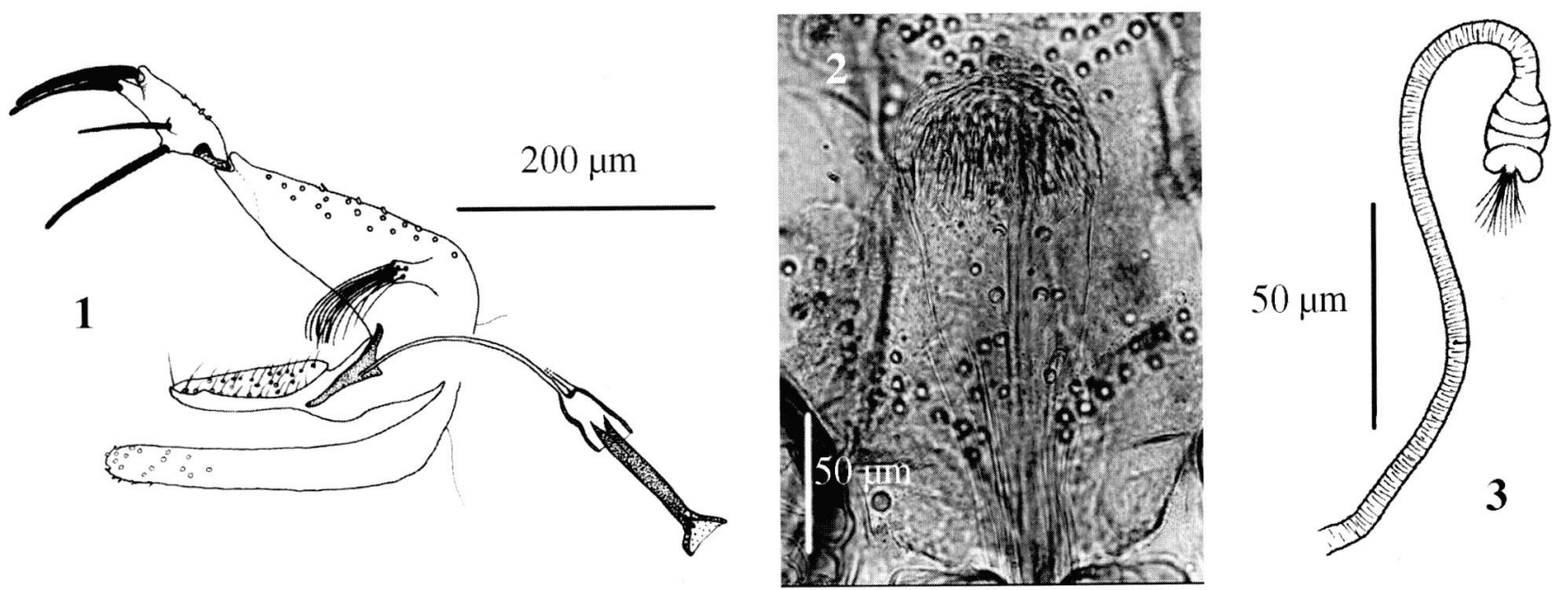

Fig. 4. - Phlebotomus sergenti. Mâle : génitalia (1). Femelle : pharynx (2) et spermathèque (3). 


\section{Phlebotomus galilaeus TheOdor, 1958 (fig. 5)}

A Chypre, elle est signalée par Adler (1946) qui la dit rare et zoophile, sous le nom de $P$. perfiliewi, la description de $P$. galilaeus en tant que sous-espèce étant postérieure à son article et le taxon n'ayant été élevé au rang d'espèce qu'en 1984 par Artemiev et Neronov.

La diagnose différentielle entre les deux espèces repose essentiellement chez les mâles sur la forme de l'extrémité des valves péniennes et sur la formule antennaire : $2 /$ III-XV chez $P$. perfiliewi et 2 /III-VIII, 1/IX-XV chez $P$. galilaeus (Artemiev \& Neronov, 1984) (en fait, nous avons noté à Chypre une certaine variabilité de ce caractère, le premier segment présentant un seul ascoïde étant tantôt le VIII, tantôt le IX).

Chez les femelles, la différence, qui porte sur la dilatation basale du conduit des spermathèques, plus allongée chez $P$. galilaeus, est plus difficile à apprécier. A notre avis, chez cette dernière espèce, l'allongement du "bonnet phrygien" est post-traumatique suite à l'intrusion des valves pointues du pénis ce qui explique son inconstance.

L'aire de répartition de $P$. galilaeus est réduite: Chypre, Jordanie (Kamhawi et al., 1995), Palestine (Theodor, 1958), Syrie (observation personnelle) et Turquie (Artemiev \& Neronov, 1984).

\section{Phlebotomus tobit Adler \& Theodor, 1930 (fig. 6)}

Cette espèce a été trouvée parasitée par Leishmania infantum zymodème MON 1 (Léger et al., 2000b) ce qui en fait le vecteur présumé de la leishmaniose canine à Chypre.

Abondante autour des chenils, elle s'attaque peu à l'Homme : nous ne l'avons jamais capturée à la piqûre. Ceci est à rapprocher du nombre peu élevé de cas humains de leishmaniose répertoriés dans l'île.

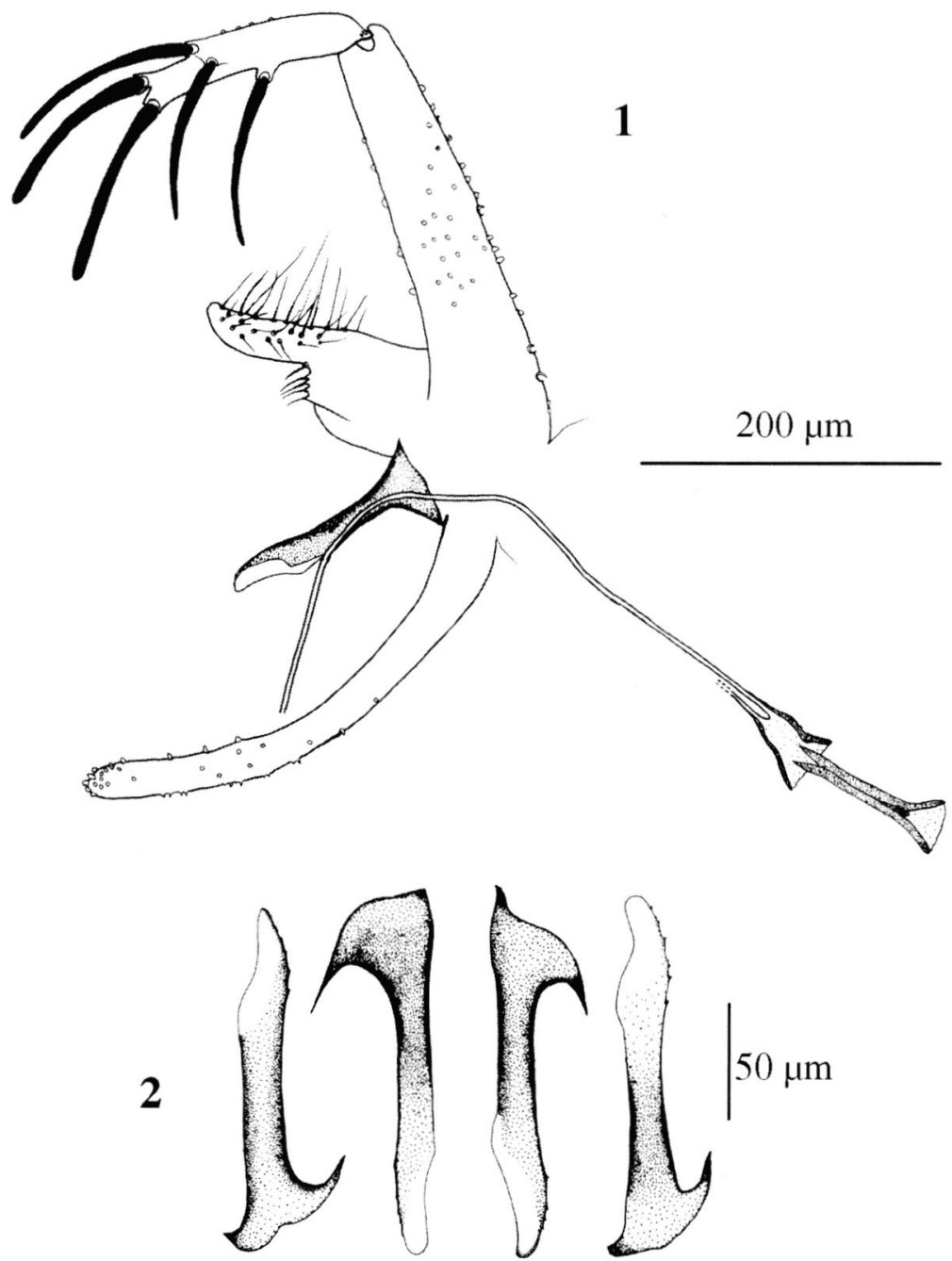

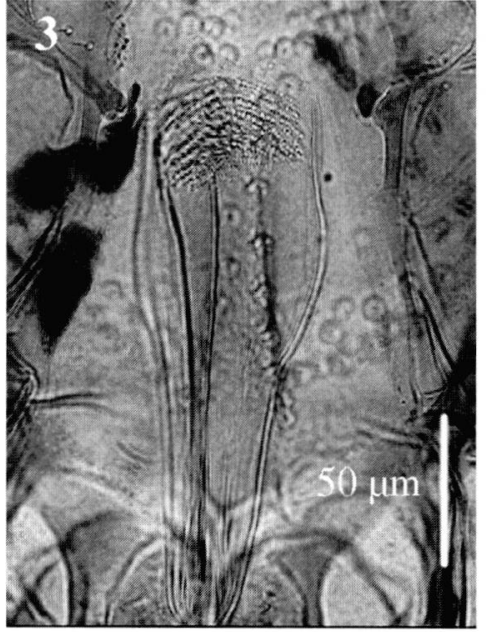

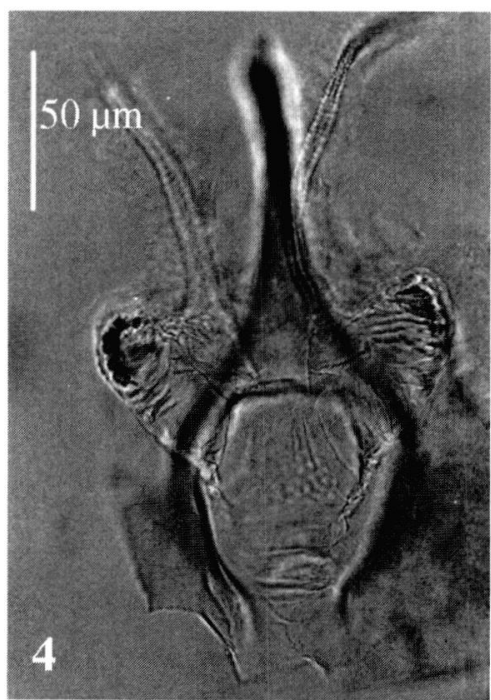

Fig. 5. - Phlebotomus galilaeus. Mâle : génitalia (1), édéages (2). Femelle : pharynx (3) et dilatation basale des spermathèques (4). 

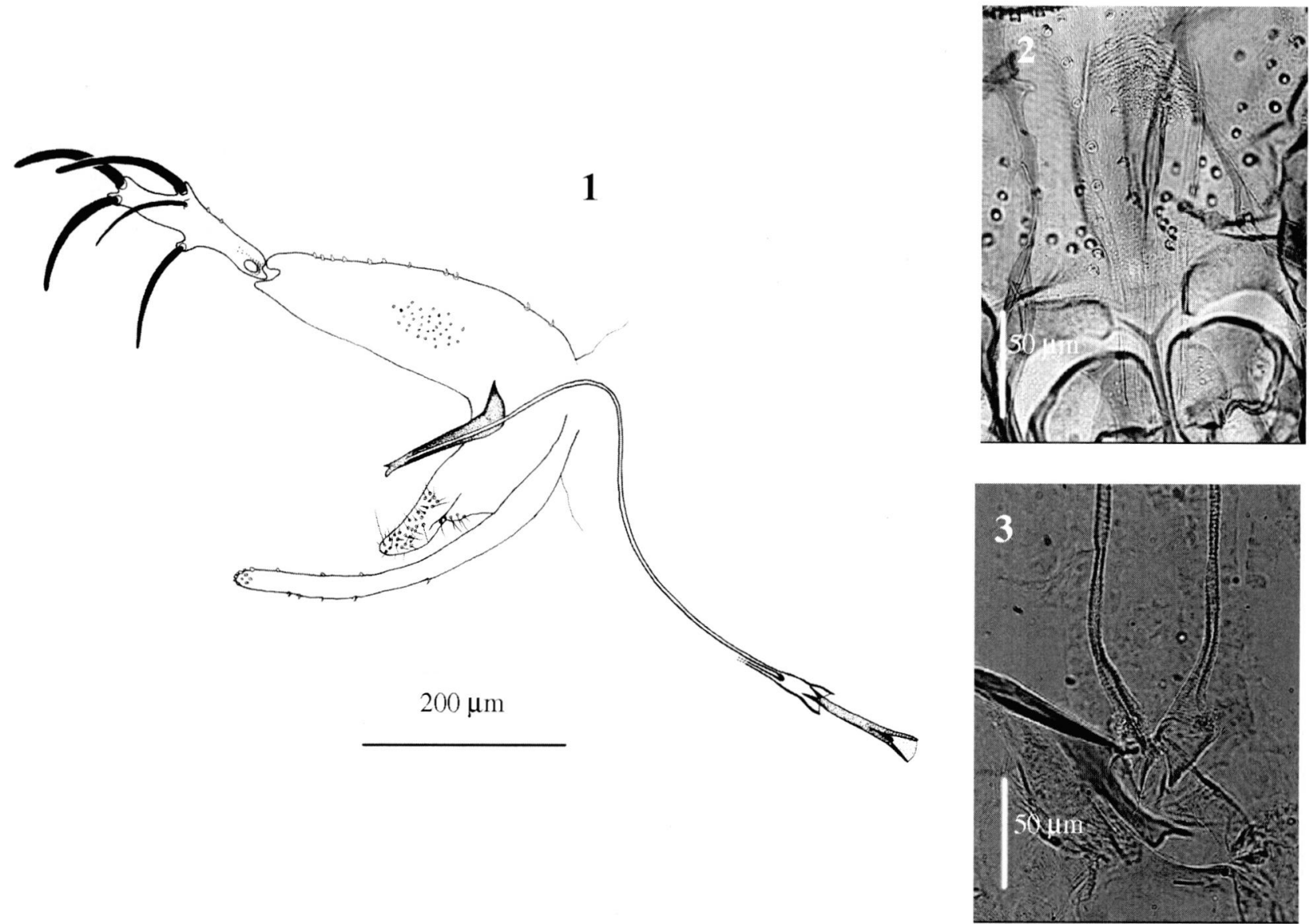

Fig. 6. - Phlebotomus tobbi. Mâle : génitalia (1). Femelle : pharynx (2) et dilatation basale des spermathèques (3).

Sur le plan morphologique, nous avons noté sur les femelles une particularité de la base des conduits des spermathèques : la courbure plus accusée de la face externe par rapport à ce qu'on observe en général dans les populations grecques. Cette légère différence, que nous considérons pour le moment de niveau populationnel, existe aussi dans les populations de Rhodes et du sud de la Macédoine.

\section{Phlebotomus kyreniae Theodor, 1958}

Mentionnée par Adler (1946) uniquement sur la côte kyrénienne, nous ne l'avons pas retrouvée durant nos enquêtes.

\section{Phlebotomus economidesi Léger, Depaquit} \& Ferté, 2000

Nous avons donné la description de cette espèce dans la première partie de ce travail ainsi que sa diagnose différentielle avec $P$. mascittii et $P$. canaaniticus (Léger et al., 2000a). Sa distribution semble relativement large. Outre son locus classicus (grotte de Mandria), elle est retrouvée à Armenochori (au nord-est de Limassol) et à Agios Georgios (entre Limassol et Paphos), tant dans des biotopes sauvages que dans des fermes.

\section{PhleBotomus masctTtil Grassi, 1908}

Adler (1946) signale la capture d'un mâle de $P$. larroussei (ultérieurement mis en synonymie avec $P$. mascittii par Theodor en 1958) à Aghirda (zone turque). Bien que la diagnose différentielle de $P$. mascittii et de $P$. canaaniticus, basée uniquement sur des critères biométriques soit délicate, Artemiev \& Neronov (1984) en font deux espèces distinctes, la seconde étant celle que l'on trouve en Israël (Adler \& Theodor, 1931), en Jordanie (Perfiliev, 1968), au Liban (Parrot \& Martin, 1944; Haddad, 1998) et en Syrie (Theodor, 1958). En se basant sur cette répartition géographique, l'espèce présente à Chypre serait plutôt $P$. canaaniticus.

En 1998, nous retrouvions à Armenochori une femelle typique de $P$. mascittii (ou P. canaaniticus?), facile à différencier de $P$. economidesi au vu de sa spermathèque en boudin plissé et de la partie initiale du conduit, renflée en vessie.

\section{SERGENTOMYIA AZIZI (ADLER, 1946) (fig. 7)}

Décrite de Chypre par Adler (1946), elle y est retrouvée par Minter et Eitrem (1989) qui, doutant de sa validité, préfèrent faire référence à Sergentomyia groupe anten- 


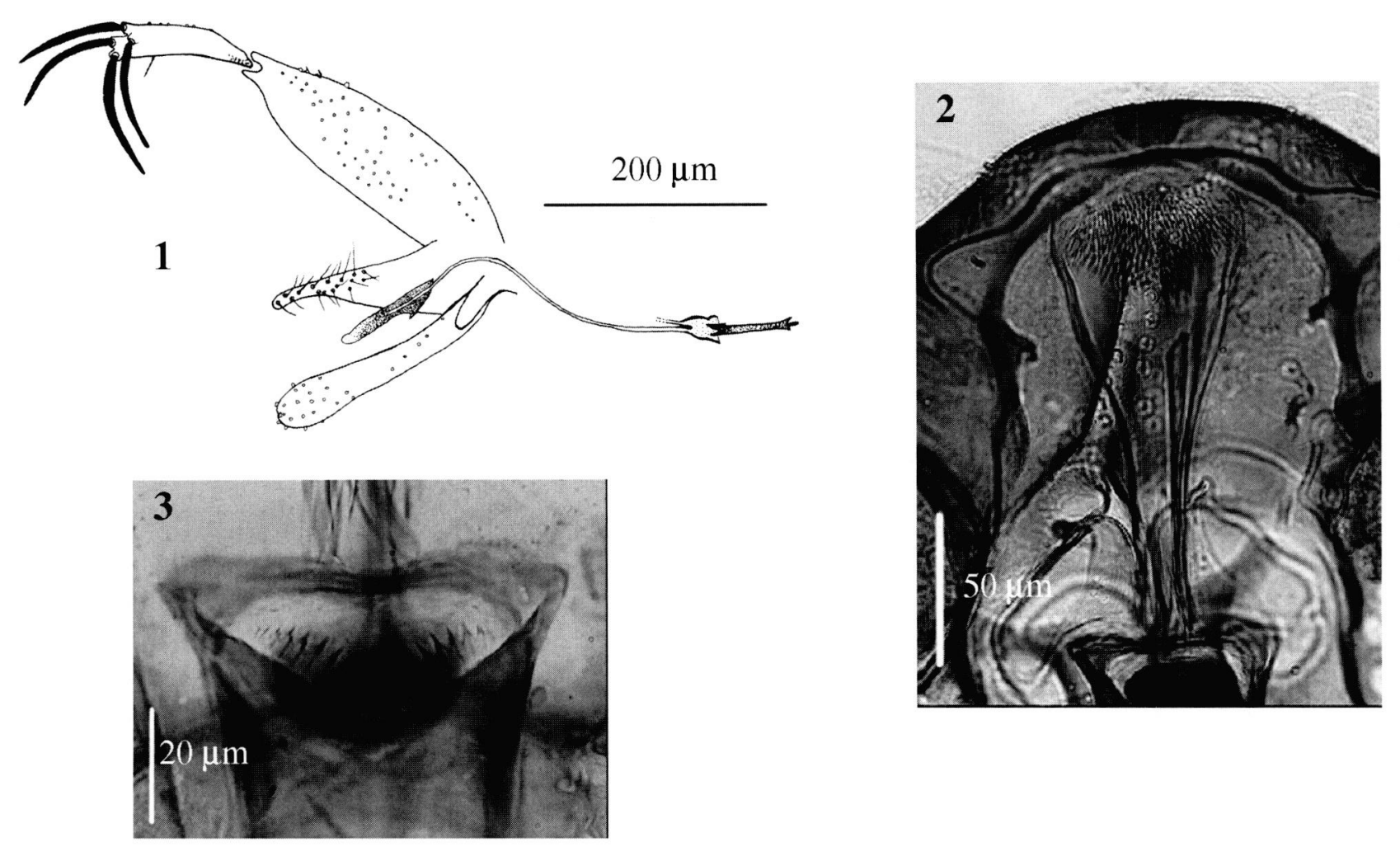

Fig. 7. - Sergentomyia azizi. Mâle : génitalia (1). Femelle : pharynx (2) et cibarium (3).

nata. Suite à une étude de Benabdennbi (communication personnelle) et à nos propres observations, nous rétablissons ici la validité du binôme $S$. azizi :

1) sur des critères morphologiques observables chez la femelle (le mâle ressemble beaucoup à celui de $S$. fallax dont il se distingue essentiellement par le style plus trapu et la position moins distale de sa soie non caduque, parfois difficile à apprécier) :

- dents cibariales subégales (alors que chez $S$. dentata, les médianes sont nettement plus petites), en moyenne (26) plus nombreuses (22 à 33 dans nos captures, 30 à 35 pour Theodor (1958) et 24 à 32 pour Benabdennbi (communication personnelle)) que chez $S$. dentata (16 à 35),

- surface pigmentée du cibarium large et triangulaire, - pharynx relativement étroit avec des dents relativement espacées.

2) sur des critères enzymatiques valables pour les deux sexes : présence d'un allèle spécifique au locus de la PGI (Benabdennbi, communication personnelle).

Les résultats obtenus par Aransay et al. (1999) sur des données moléculaires sont difficiles à interpréter sur la base de leur échantillonnage. Quant au travail d'Aransay et al. (2000), les séquences obtenues ne sont pas assez informatives pour résoudre les relations phylogénétiques entre $S$. dentata de Céphalonie et $S$. dentata (= S. azizi) de Chypre.
SERGENTOMYIA FALLAX (PARROT, 1921) (fig. 8)

Signalée dans les trois inventaires, cette espèce est désignée par Adler, qui est par ailleurs le premier à donner une description de la femelle, sous le trinôme P. fallax cypriotica.

Nous avons comparé nos captures cypriotes à celles provenant de diverses régions voisines : Grèce, Liban, Syrie et plus lointaines : Maroc. Les variations observées (nombre de dents cibariales, rapport coxite/style) restent de niveau individuel et le statut de sous-espèce (ou, selon Adler, de variété) nous semble injustifié.

\section{SERGENTOMYIA MINUTA (RONDANI, 1843) (fig. 9)}

Etudiée par Benabdennbi (communication personnelle), la population cypriote de $S$. minuta ne montre aucune particularité par rapport aux autres populations méditerranéennes. En particulier le nombre moyen de dents cibariales chez la femelle s'inscrit à la place attendue dans le cline nord-sud précédemment mis en évidence (Rioux et al., 1975; Léger et al., 1979; Belazzoug et al., 1982; Pesson et al., 1984) (Fig. 10) ce qui, du même coup, fait tomber en synonymie $P$. parroti (binôme utilisé par Adler en 1946) avec S. minuta. 

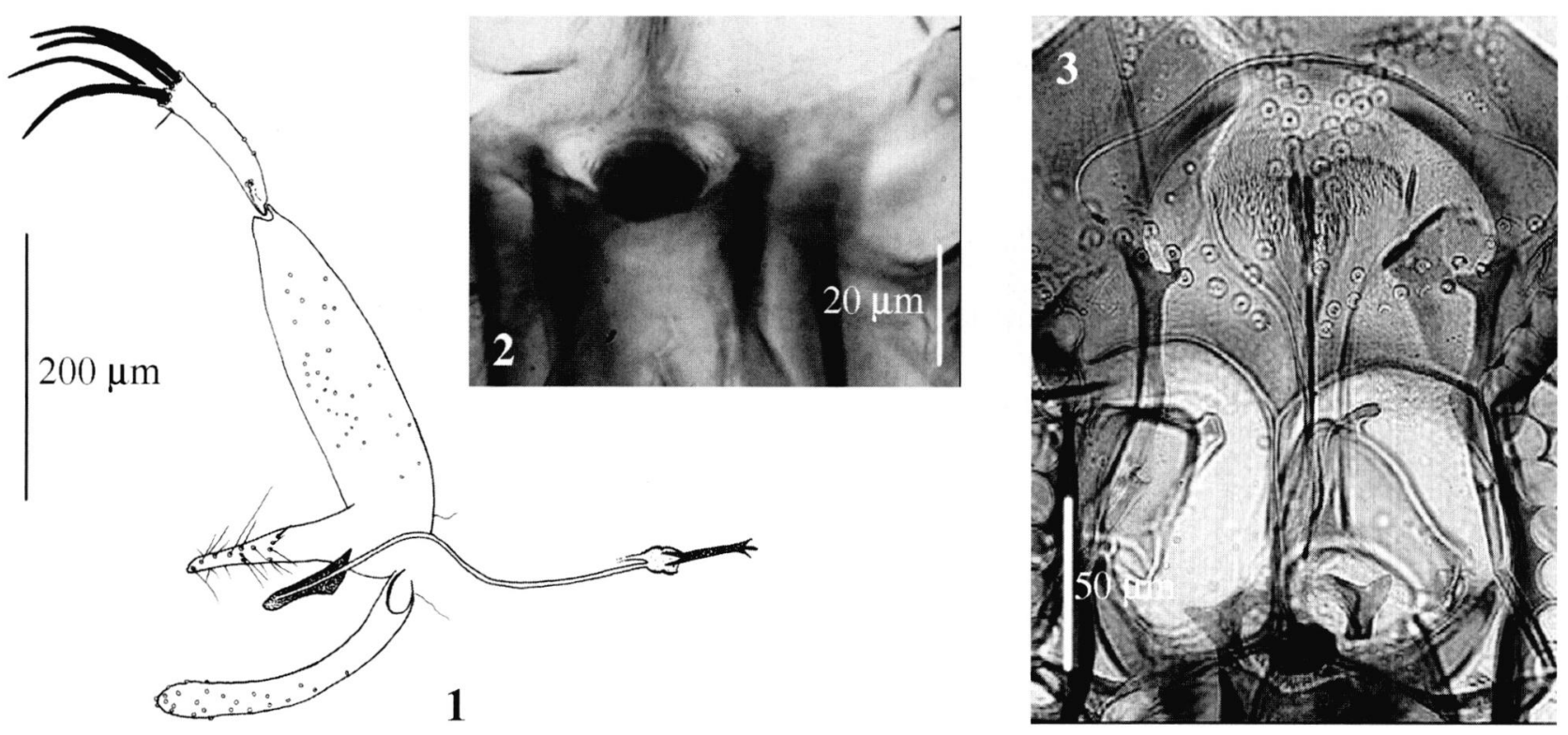

Fig. 8. - Sergentomyia fallax. Mâle : génitalia (1). Femelle : cibarium (2) et pharynx (3).
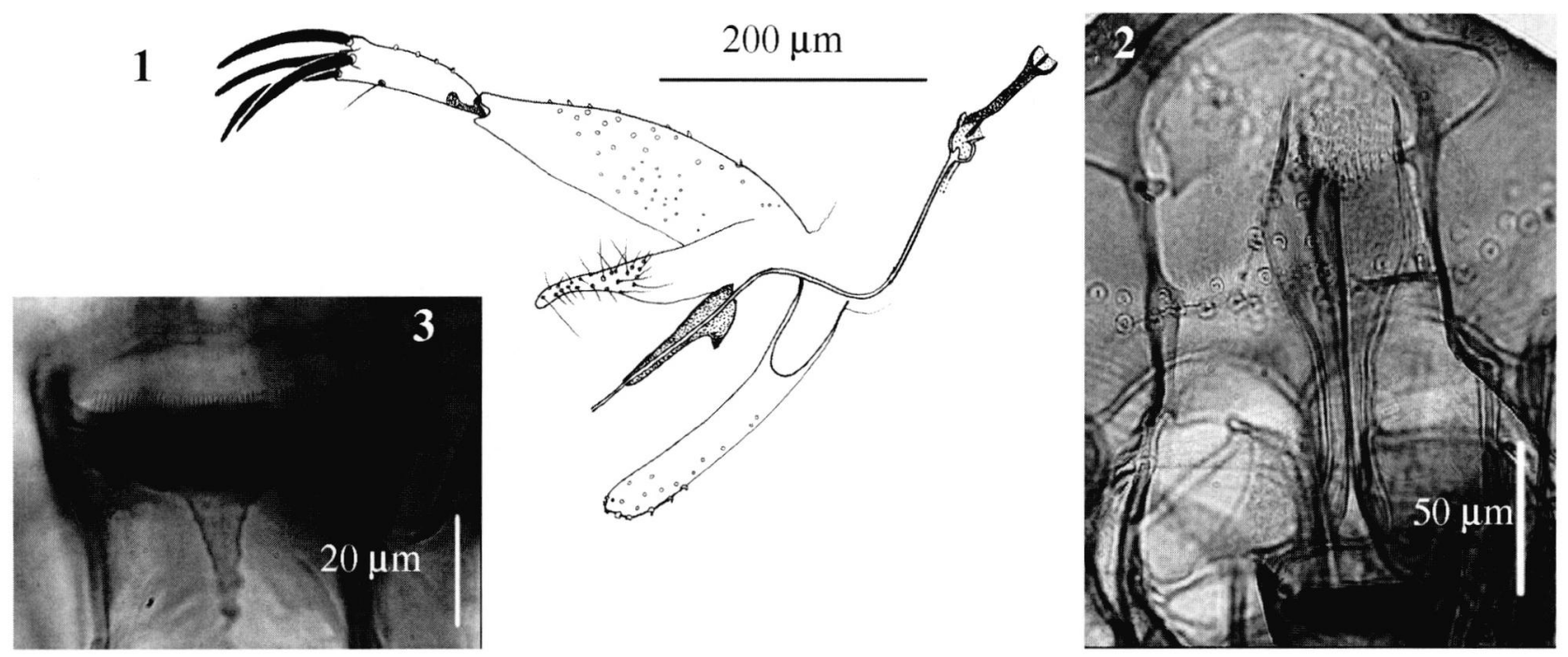

Fig. 9. - Sergentomyia minuta. Mâle : génitalia (1). Femelle : pharynx (2) et cibarium (3).

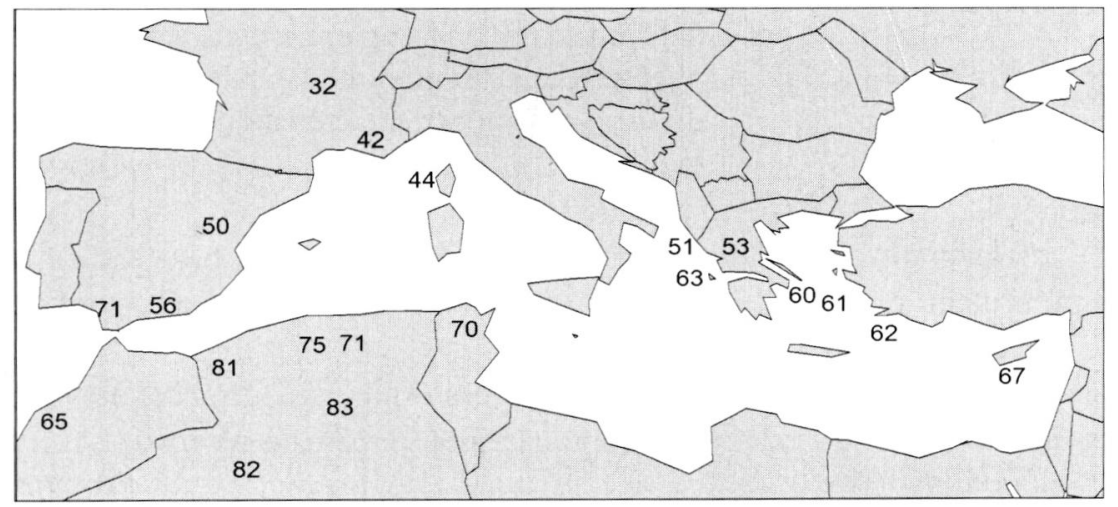

Fig. 10. - Carte représentant les valeurs moyennes du nombre de dents cibariales de S. minuta dans différentes régions du bassin méditerranéen (d'après Benabdennbi, Étude de populations de Sergentomyia (Psychodidae, Phlebotominae) de l'est méditerranéen. Mémoire de DEA interactions hôtesparasites, Paris XII, 1994). 


\section{CLÉ D'IDENTIFICATION DES PHLÉBOTOMES DE CHYPRE}

MÂLES

Quatre épines sur le style

- terminales ou 2 terminales et 2 subterminales. Pas de lobe basal du coxite

- édéage effilé, 1 soie non caduque sur le style, située à environ la moitié de la longueur. S. minuta

- édéage épais, arrondi à l'extrémité (diagnose spécifique parfois délicate)

- style avec 2 épines terminales et 2 légèrement décalées; insertion de la soie caduque située environ au tiers distal S. $a z i z i$

- coxite et style grêles, ce dernier avec 4 épines terminales; insertion de la soie non caduque juste audessous d'elles S. fallax

- 2 terminales (ou 1 terminale et 1 subterminale) et deux médianes

- présence d'un lobe basal du coxite garni de soies plus ou moins longues

- AIII $\leq 160 \mu \mathrm{m}$. Style avec une épine terminale portée par un long pédoncule. Coxite portant un lobe basal en "pomme d'arrosoir " P. alexandri

. AIII $>200 \mu \mathrm{m}$

style avec 2 épines terminales; une des deux médianes souvent plus grêle que l'autre; lobe basal du coxite étroit et arqué avec une quinzaine de soies implantées sur l'extrémité légèrement renflé P. sergenti épine terminale du style sur un pédoncule, nettement décalée par rapport à l'épine subterminale; lobe basal du coxite droit et épais portant environ 25 soies P. jacusieli

- absence de lobe basal du coxite......P. economidesi ${ }^{*}$

Cinq épines sur le style

- paramère trilobé. Un petit lobe basal sur le coxite... P. papatasi

- paramère simple. Pas de lobe basal sur le coxite

- édéage présentant une extrémité caractéristique

- extrémité fourchue P. tobbi

- extrémité spatulée translucide $P$. galilaeus

- extrémité munie d'un tubercule subterminal P. kyreniae

- édéage arrondi à son extrémité ne présentant ni fourche, ni tubercule subterminal

- édéage régulièrement atténué P. mascittii - édéage légèrement rétréci à sa base et un peu dilaté au niveau du tiers distal P. economidesi"
Six épines sur le style P. economidesi

* : P. economidesi présente un nombre variable d'épines au niveau du style : cinq ou six, parfois quatre.

\section{FeMelles}

Cibarium armé

- une soixantaine de dents disposées en palissade sur une bande pigmentaire bien développée; pharynx peu armé, garni d'épines

S. minuta

- dents pointues disposées en arc de cercle avec une plage pigmentée centrale

- 22 à 35 dents subégales; pharynx légèrement cordiforme armé d'une seule catégorie de dents....S. azizi

- 14 à 20 dents égales; pharynx nettement cordiforme, très large, armé de deux séries bien tranchées de dents

S. fallax

Cibarium inerme

- spermathèque annelée

- à tête portée sur un long cou (Larroussius) et dont la base des conduits présente une dilatation caractéristique

. en cloche asymétrique P. tobbi

- en bonnet phrygien P. galilaeus

- à tête sessile

- tête en pompon rond; anneaux de la spermathèque d'égale épaisseur; armature pharyngienne formée de peignes

P. papatasi

. tête petite, invaginée dans l'anneau terminal, souvent plus épais que les autres; armature pharyngienne formée d'écailles pointues

AIII $\leqslant 160 \mu \mathrm{m} ;$ spermathèque comportant 7 à 10 anneaux; armature pharyngienne rectangulaire en réseau P. alexandri

AIII $>200 \mu \mathrm{m}$

spermathèque comportant 4 à 6 anneaux; écailles de l'armature pharyngienne longues, en flammes de bougie, disposées en bulbe d'oignon sur toute la largeur du pharynx .P. sergent

spermathèque comportant 8 à 9 anneaux; écailles de l'armature pharyngienne plus épaisses et plus serrées au centre que latéralement, offrant un aspect compact caractéristique de la partie médiane P. jacusieli

- spermathèque plissée

- conduit étroit avec un épaississement basal.

P. kyreniae

- conduit large

- plissé de diamètre uniforme P. economidesi

- présentant une dilatation proximale en vessie 


\section{COMMENTAIRE BIOGÉOGRAPHIQUE}

I

'absence à Chypre de $P$. neglectus, vecteur de Leishmania infantum en Grèce continentale et insulaire, et de son vicariant $P$. syriacus, qui le remplace au Proche-Orient (versant sud-est du Taurus, Syrie, Liban, Jordanie, Israël), ainsi que la présence d'un Transphlebotomus (P. economidesi) et d'un Sergentomyia $(S$. azizi) endémiques suggèrent des voies de peuplement originales à partir du Miocène, interrompues par de longues périodes d'isolement.

Actuellement, l'île est formée de quatre zones géologiques disposées en bandes parallèles du rivage kyrénien à la façade ouest méditerranéenne (Panayiotou, 1983) (Fig. 11) :

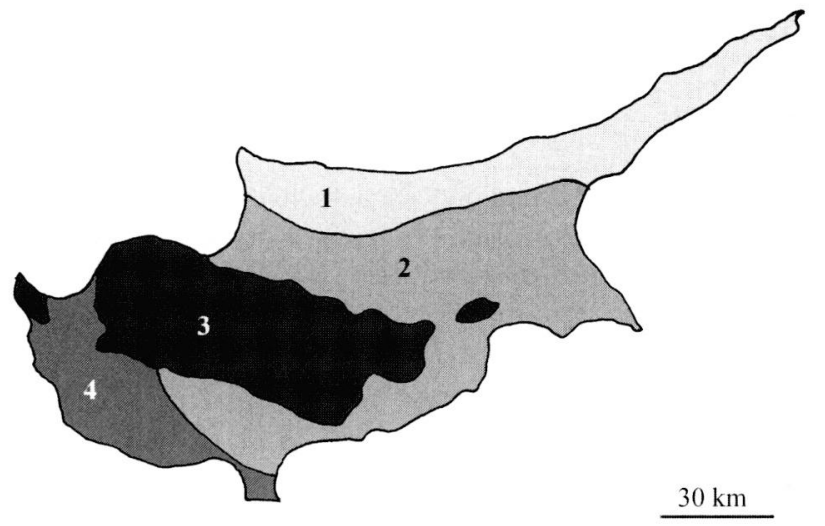

Fig. 11. - Carte géologique (d'après la carte établie par the Geological Survey Department, Ministry of Agriculture, Natural Resources and Environment, Cyprus, 1995). Terranes kyréniens (1), couches sédimentaires entourant le Troodos (2), ophiolithes du Troodos (3) et terranes de la Mamonia (4).

- les terranes de la bordure kyrénienne formés de dépôts qui se sont entassés depuis le Permien jusqu'au milieu du Miocène, où les reliefs, suite de collines culminant entre 700 et 1024 mètres, sont considérés comme l'ultime avancée méridionale de la ceinture alpine taurodinarique,

- les vallées, notamment la plaine de la Mésorée, qui entourent le massif du Troodos, dans lesquelles les sédiments se sont déposés en couches successives entre le Crétacé supérieur et le Pleistocène,

- les ophiolithes du Troodos, de nature volcanique, accumulés au fond de la Téthys durant le Crétacé supérieur, le début de l'émergence du massif se situant au Miocène inférieur et se continuant jusqu'au début du Pleistocène avec une poussée finale atteignant 2000 mètres d'altitude,

- les terranes de Mamonia qui datent du Trias et du Crétacé et dont la mise en place actuelle et la soudure au Troodos se seraient faites du Crétacé moyen au Maas- trichtien (Crétacé supérieur) le long d'une zone de subduction sous le Troodos, lors de la collision des plaques afro-arabique et eurasienne qui entraîna également une rotation de $90^{\circ}$ de la microplaque du Troodos. Le climat très varié de l'île engendre une grande profusion d'habitats et une diversité floristique et faunistique exceptionnelles. L'endémisme atteint un haut niveau (environ 110 espèces végétales). La dualité zone kyrénienne et Troodos-Mamonia s'y retrouve, témoignant de l'émergence tardive de la plaine de la Mésorée, recouverte par la mer depuis l'ouverture du détroit de Gibraltar il y a environ 5 millions d'années jusqu'au Pléistocène (1,6 millions d'années).

Ainsi, nous n'avons pas trouvé dans la partie grecque de l'île, la seule que nous ayons prospectée, $P$. (Adlerius) kyreniae Theodor, 1958, signalé sous le nom de P. chinensis par Adler (1946) dans la partie actuellement turque de l'île.

L'histoire géologique de Chypre, dont le début de l'émergence date du Miocène inférieur, fait apparaître deux périodes possibles de peuplement. La première se situe au cours de la crise messinienne (assèchement de la Téthys au Miocène), période active d'échanges entre les zones exondées. C'est également une période de différenciation spécifique pour les Phlébotomes méditerranéens dont les sous-genres sont déjà en place (Léger \& Pesson, 1987; Pesson et al., 1994). La seconde prend place au Pléistocène où, durant les épisodes glaciaires, le nord-est de l'île (cap Andreas) se trouvait en relation avec le nord de la Syrie par l'intermédiaire d'un véritable pont d'îlots utilisé par les premiers mammifères de l'île, éléphants et hippopotames qui, à la faveur de l'isolement des périodes interglaciaires entraînant une élévation du niveau de la mer, donnèrent naissance à des espèces naines (Attenborough, 1987).

Ces échanges privilégiés avec le nord de la Syrie sont peut-être illustrés dans notre travail par le fait que c'est à partir de $P$. tobbi, vecteur inhabituel, que nous avons isolé des leishmanies à la fois à Chypre (Léger et al., 2000 b) et dans la région de Kassab, au nord de la Syrie (Rioux et al., 1998).

Reste le problème posé par l'absence à Chypre de P. syriacus, abondant en Syrie. Cette espèce étant plus thermophile que $P$. tobbi, nous pensons que son aire de répartition a connu des régressions lors des périodes glaciaires au cours desquelles justement le contact Chypre-Syrie était établi, ce qui expliquerait qu'elle ne soit pas passée.

\section{REMERCIEMENTS}

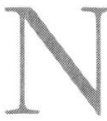

ous remercions messieurs Symeon Matsis (Permanent Secretary, Ministry of Agriculture, Natural Resources and Environment, Cyprus), 
Savvas Theophanous (Director, Department of Forest, Ministry of Agriculture, Natural Resources and Environment, Cyprus) and Christos Alexandrou (Director, Forestry College, Ministry of Agriculture, Natural Resources and Environment, Cyprus) pour leur hospitalité et leur assistance qui ont rendu cette étude possible.

\section{RÉFÉRENCES}

AdLer S. The sandflies of Cyprus (Diptera). Bulletin of Entomological Research, 1946, 36, 497-511.

Adler S. \& Theodor O. Investigations on Mediterranean Kala Azar. III. The sandflies of the Mediterranean Basin. Distribution and Bionomics of Sandflies in Catania and District. Proceedings of the Royal Society (B), 1931, 108, 464-480.

Aransay A.M., Scoulica E., Chaniotis B. \& Tselentis Y. Typing of sandflies from Greece and Cyprus by DNA polymorphism of 185 rRNA gene. Insect Molecular Biology, 1999, 8, 179-184.

Aransay A.M., Scoulica E., Tselentis Y. \& Ready P.D. Phylogenetic relationships of Phlebotomine sandflies inferred from small subunit nuclear ribosomal DNA. Insect Molecular Biology, 2000, 9, 157-168.

Artemiev M. \& Neronov V. Distribution and ecology of sandflies of the world (genus Phlebotomus). Inst. Evol. Morph. Anim. Ecol., Moscou, USSR, 1984, 208 p.

Attenborough D. The first Eden. The Mediterranean world and Man. Collins Sons \& Co. Ltd Eds, Glasgow, Great Britain, 1987, $240 \mathrm{p}$.

Belazzoug S., Mahzoul D., Addadi K. \& Dedet J.P. Sergentomyia minuta parroti (Adler \& Theodor, 1927) en Algérie (Diptera, Psychodidae). Annales de Parasitologie Humaine et Comparée, 1982, 57, 621-630.

Depaquit J., Ferté H., Léger N., Killick-Kendrick R., Rioux J.A., Killick-Kendrick M., Hanafi H.A. \& Gobert S. Molecular systematics of the Phlebotomine sandflies of the subgenus Paraphlebotomus (Psychodidae, Diptera, Phlebotomus) based on ITS 2 rDNA sequences. Hypotheses of dispersion and speciation. Insect Molecular Biology, 2000, 9, 293-300.

Depaqutt J., LÉGer N. \& Ferté H. Le statut taxinomique de Phlebotomus sergenti Parrot, 1917, vecteur de Leishmania tropica (Wright, 1903) et Phlebotomus similis Perfiliev, 1963 (Diptera - Psychodidae). Approches morphologique et morphométrique. Corollaires biogéographiques et épidémiologiques. Bulletin de la Société de Pathologie Exotique, 1998, 91, 346-352.

HADDAD N. Les Phlébotomes du Liban. Inventaire et corollaire éco-épidémiologique. Thèse d'Université, Reims, 1998, $\mathrm{n}^{\circ} 208,220$ p.

Kamhawi S.A., Abdel-Hafez S.K. \& Molyneux D.H. A comprehensive account of species composition, distribution and ecology of phlebotomine sandflies in Jordan. Parasite, $1995,2,163-172$.

Léger N., Saratsiotis A., Pesson B. \& Léger P. La leishmaniose en Grèce. Résultats d'une enquête entomologique effec- tuée en juin 1977. Annales de Parasitologie Humaine et Comparée, 1979, 54, 11-29.

LÉGER N. \& Pesson B. Sur la taxinomie et la répartition géographique de Phlebotomus (Adlerius) chinensis s.l. et de $P$. (Larroussius) major s.l. (Psychodidae, Diptera). Statut des espèces présentes en Grèce. Bulletin de la Société de Pathologie Exotique, 1987, 80, 252-260.

Léger N., Depaquit J. \& Ferté H. Les Phlébotomes de l'île de Chypre. I. Description de Phlebotomus (Transphlebotomus) economidesi n. sp. Parasite, 2000a, 7, 135-141.

Léger N., Depaquit J., Ferté H., Rioux J.A., Gantier J.C., Gramiccia M., Ludovisi A., Michaelides A., Christophi N. \& ECONOMIDES P. Les Phlébotomes de l'île de Chypre. II. Présence de Leishmania (Leishmania) infantum Nicolle, 1908 (zymodème MON 1) chez Phlebotomus (Larroussius) tobbi Adler et Theodor, 1930. Parasite, 2000b, 7, 143-146.

Minter D. \& Eitrem U. Sandflies and disease in Cyprus; 1944 1985, in : Leishmaniasis - The current status and new strategies for control. Hart D.T. (ed), Plenum press, New York, 1989, 207-216.

Panayiotou A. Geology of Cyprus. Cyprus Today, 1983, 3, $2-10$.

PARrot L. \& Martin R. Notes sur les Phlébotomes. XLII. Sur Phlebotomus larroussei var. canaaniticus. Archives de l'Institut Pasteur d'Algérie, 1944, 22, 47-51.

Perfiliev P.P. Fauna of USSR. Diptera. Phlebotomidae (sandflies). Translated by Israel Program for Scientific translation. Theodor O. (ed), 1968, 363 p.

Pesson B., Léger N. \& Madulo-Leblond G. La leishmaniose en Grèce : les Phlébotomes des Iles Ioniennes et de la mer Egée. Annales de Parasitologie Humaine et Comparée, 1984, 59, 277-296.

Pesson B., Léger N., Madulo-Leblond G., Ferté H., Tselentis I. Papadopollos B. \& Perieres J. Spéciation et vicariance chez les Phlébotomes des îles grecques (référence à Phlebotomus neglectus et au sous-genre Larroussius). Bios, 1994, 2, 233-243.

Rioux J.A., Croset H., Léger N. \& Maistre M. Remarques sur la taxonomie infraspécifique de Sergentomyia minuta, Sergentomyia africana et Sergentomyia antennata. Annales de Parasitologie Humaine et Comparée, 1975, 50, 635-641.

Rioux J.A., Léger N., Haddad N., Gramiccia M., Jalouk L., Dereure J., Al-Khiami A. \& Desjeux P. Infestation naturelle de Phlebotomus tobbi (Diptera, Psychodidae) par Leishmania donovani s. st. (Kinetoplastida, Trypanosomatidae), en Syrie. Parassitologia, 1998, 40 (Suppl. 1), 148.

Theodor O. Psychodidae-Phlebotominae. Die Fliegen der Palearktischen Region, 1958, 201, 1-55.

Reçu le 21 septembre 2000 Accepté le 29 septembre 2000 